\title{
Dynamic Nuclear Polarization with Single Electron Spins
}

\section{Citation}

Petta, J. R., J. M. Taylor, A. C. Johnson, A. Yacoby, M. D. Lukin, C. M. Marcus, M. P. Hanson, and A. C. Gossard. 2008. "Dynamic Nuclear Polarization with Single Electron Spins." Physical Review Letters 100 (6). https://doi.org/10.1103/physrevlett.100.067601.

\section{Permanent link}

http://nrs.harvard.edu/urn-3:HUL.InstRepos:41417316

\section{Terms of Use}

This article was downloaded from Harvard University's DASH repository, and is made available under the terms and conditions applicable to Other Posted Material, as set forth at http:// nrs.harvard.edu/urn-3:HUL.InstRepos:dash.current.terms-of-use\#LAA

\section{Share Your Story}

The Harvard community has made this article openly available.

Please share how this access benefits you. Submit a story.

Accessibility 


\title{
Dynamic Nuclear Polarization with Single Electron Spins
}

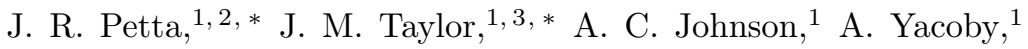 \\ M. D. Lukin, ${ }^{1}$ C. M. Marcus, ${ }^{1}$ M. P. Hanson, ${ }^{4}$ and A. C. Gossard ${ }^{4}$ \\ ${ }^{1}$ Department of Physics, Harvard University, 17 Oxford St., Cambridge, MA 02138 \\ ${ }^{2}$ Department of Physics, Princeton University, Princeton, New Jersey 08544 \\ ${ }^{3}$ Department of Physics, Massachusetts Institute of Technology, Cambridge, MA 02139 \\ ${ }^{4}$ Materials Department, University of California, Santa Barbara, California 93106
}

\begin{abstract}
We polarize nuclear spins in a GaAs double quantum dot by controlling two-electron spin states near the anti-crossing of the singlet $(\mathrm{S})$ and $m_{S}=+1$ triplet $\left(T_{+}\right)$using pulsed gates. An initialized $\mathrm{S}$ state is cyclically brought into resonance with the $T_{+}$state, where hyperfine fields drive rapid rotations between $\mathrm{S}$ and $T_{+}$, 'flipping' an electron spin and 'flopping' a nuclear spin. The resulting Overhauser field approaches $80 \mathrm{mT}$, in agreement with a simple rate-equation model. A self-limiting pulse sequence is developed that allows the steady-state nuclear polarization to be set using a gate voltage.
\end{abstract}

PACS numbers: 03.67.Bg, 73.21.La, 76.70.-r

Semiconductor quantum dots share many features with real atoms: they possess discrete electronic energy states, obey Hund's rules as the states are filled, and can be coupled to one another, creating artificial molecules [1, 2]. In contrast to atoms, where electrons are coupled to a single nucleus, electrons within quantum dots interact with many (typically $\sim 10^{6}$ ) lattice nuclei 2 . The dynamics of spin systems comprising few electrons interacting with many nuclei is an interesting and complex many-body problem in condensed matter physics [3, 4, 5, 6].

Electrically controlled nuclear polarization in semiconductor microstructures has been investigated in gatedefined quantum point contacts (QPCs) in GaAs, where nuclear polarization driven by scattering between spinpolarized edge states induced hysteresis in conductance as a function of magnetic field 7, 8. In few-electron quantum dots, transport in the so-called Pauli blockade regime, which requires a spin flip for conduction, can exhibit long-time-scale oscillations and bi-stability, also understood to result from a build-up and relaxation of nuclear polarization during spin-flip-mediated transport 6. 9, 10, 11. Depending on conditions, sizable polarizations can result from transport in this regime [12]. Spin relaxation at low magnetic fields, (time scale $T_{1}$ ) and spin dephasing (time scale $T_{2}^{*}$ ) in GaAs double quantum dots are limited by hyperfine interactions with host nuclei [13, 14, 15, 16, 17. It has been suggested that electron spin dephasing times may be extended by cooling nuclear spins through nuclear polarization and projective measurement [6, 18, 19. In addition, nuclear spins may be used as a quantum memory due to long nuclear spin coherence times [20. For these reasons, it is desirable to have the ability to control interactions between a single confined electron spin and a bath of quantum dot nuclear spins [21].

In this Letter, we use fast pulsed-gate control of twoelectron spin states in a double quantum dot to create and detect nuclear polarization. Rather than using spin-
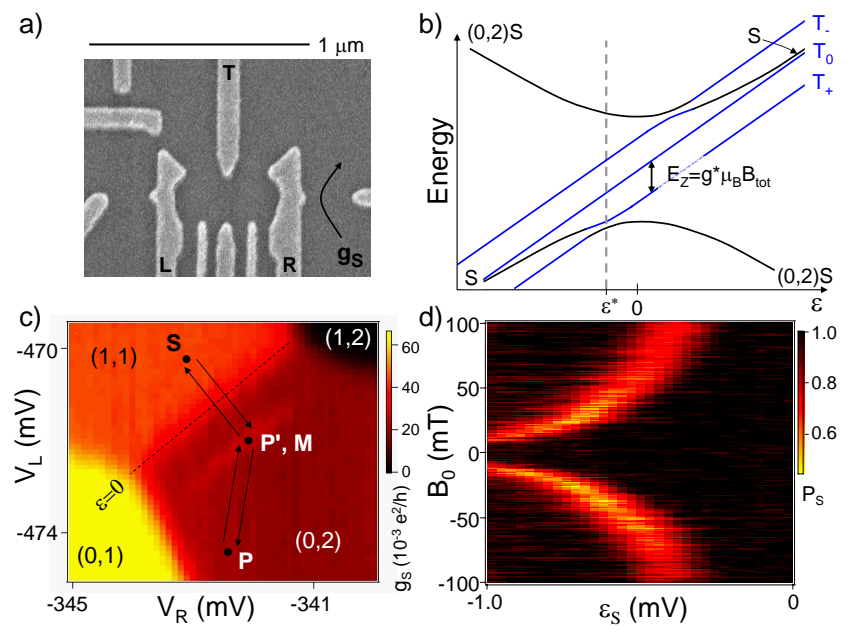

FIG. 1: (a) SEM image of a device similar to the one used in this experiment. Gates L, R set the occupancy of the double dot while gate $\mathrm{T}$ tunes the interdot tunnel coupling. A QPC with conductance, $g_{\mathrm{S}}$, senses charge on the double dot. (b) Charge sensor conductance, $g_{\mathrm{S}}$, measured as a function of $V_{L}$ and $V_{R}$. A pulse sequence used to polarize nuclei is superimposed on the charge stability diagram (see text). The bright signal at point $M$ that runs parallel to the $\epsilon=0$ line is a result of Pauli-blocked $(1,1) \rightarrow(0,2)$ charge transitions and reflects $S-T_{+}$mixing at point $\mathrm{S}$. A background plane has been subtracted from the data. (d) Singlet return probability $P_{S}$ for separation time $\tau_{\mathrm{S}}=200 \mathrm{~ns}$, as a function of separation position $\epsilon_{\mathrm{S}}$ and applied field $B_{0}$. The field dependent "spin funnel" corresponds to the $S-T_{+}$anti-crossing position, $\epsilon_{\mathrm{S}}=\epsilon^{*}$.

flip-mediated transport with an applied bias [11, 12, we use a cycle of gate voltages to prepare a spin singlet $(S)$ state, induce nuclear flip-flop at the singlet-triplet $\left(S-T_{+}\right)$ anti-crossing, and measure the resulting nuclear polarization from the position of that anti-crossing. In this way, dynamic nuclear polarization (DNP) is achieved (and measured) by the controlled flipping of single electron spins. Additionally, we demonstrate a self-limiting pulse 
cycle with fixed period that allows voltage-controlled "programming" of steady-state nuclear polarization. Experimental results are found to be in qualitative agreement with a simple rate-equation model.

Measurements are carried out using a gated-defined GaAs double quantum dot [14]. The device is configured near the (1,1)-(0,2) charge transition (see Fig. 1(a)). A QPC charge sensor is used to read out the time-averaged charge configuration of the dot, from which spin states can be inferred. Relevant energies of spin states as a function of detuning, $\epsilon$, are shown in Fig. 1(b). The $(1,1)$ singlet, $S$, and $(0,2)$ singlet, denoted $(0,2) S$, hybridize near $\epsilon=0$ due to interdot tunneling. Triplets of $(0,2)$ are not considered, due to the large singlet-triplet splitting, $E_{S T} \sim 400 \mu \mathrm{eV}$. The triplet states of $(1,1), T_{+}$, $T_{-}$, and $T_{0}$, do not hybridize with $(0,2) S$ due to spin selection rules. An external magnetic field, $\vec{B}_{0}$, applied perpendicular to the plane of the two-dimensional electron gas (2DEG), adds to the hyperfine field, $\vec{B}_{\text {nuc }}$, resulting in a total effective Zeeman field $\vec{B}_{\text {tot }}=\vec{B}_{0}+\vec{B}_{\text {nuc }}$. The Zeeman energy, $E_{\mathrm{Z}}=g^{*} \mu_{\mathrm{B}} B_{\text {tot }}$, lifts the triplet-state degeneracy, where $B_{\text {tot }}=\left|\vec{B}_{\text {tot }}\right|$ and $g^{*} \sim-0.4$ is the effective electronic g-factor in GaAs. The nuclear Zeeman field is proportional to nuclear polarization and has a magnitude $\left|\vec{B}_{\text {nuc }}\right|=5.2 \mathrm{~T}$ for fully polarized nuclei in GaAs $[22]$.

Hyperfine fields rapidly drive transitions between $S$ and $T_{0}$ on a 10 ns time scale at large negative detuning, where these states become degenerate [10, 14]. However, transitions between $S$ and $T_{0}$ do not polarize nuclei since there is no change in the total spin component along the field, $\Delta m_{S}=0$. In contrast, the transition from $S$ to $T_{+}$ involves a change in electron spin, $\Delta m_{S}=1$. When driven by the hyperfine interaction, this electron spin 'flip' is accompanied by a nuclear spin 'flop' with $\Delta m_{I}=-1$. The change in $B_{\text {nuc }}$ associated with this flip-flop transition is along the direction of external field, taking into account the positive nuclear $\mathrm{g}$ factors for $\mathrm{Ga}$ and $\mathrm{As}$ [2]. The cyclic repetition of the transition from $S$ to $T_{+}$can thereby lead to a finite time-averaged $B_{\text {nuc }}$ oriented along the external field.

The value of detuning where the $S-T_{+}$anti-crossing occurs, denoted $\epsilon^{*}$ (see Fig. 1(b)), is a sensitive function of $B_{\text {tot }}$ for $\left|B_{\text {tot }}\right| \leq 80 \mathrm{mT}$, providing a straightforward means of measuring $B_{\text {nuc }}$ within that range. To calibrate the measurement, the dependence of $\epsilon^{*}$ on external field amplitude $B_{0}$ is measured using the pulse sequence shown in Fig. 1(c): The $(0,2) S$ state is first prepared at point P. A delocalized singlet in $(1,1)$ is created by moving to point $\mathrm{S}$ (detuning $\epsilon_{\mathrm{S}}$ ) via point $\mathrm{P}^{\prime}$. The system is held at point $S$ for a time $\tau_{S} \gg T_{2}^{*}$ then moved to point $M$ and held there for the longest part of the cycle. The sequence is then repeated. When $\epsilon_{\mathrm{S}}=\epsilon^{*}$, rapid mixing of $S$ and $T_{+}$ states occurs. When the system is moved to point $M$, the $(1,1)$ charge state will return to $(0,2)$ only if the separated spins are in a singlet configuration. The probability of
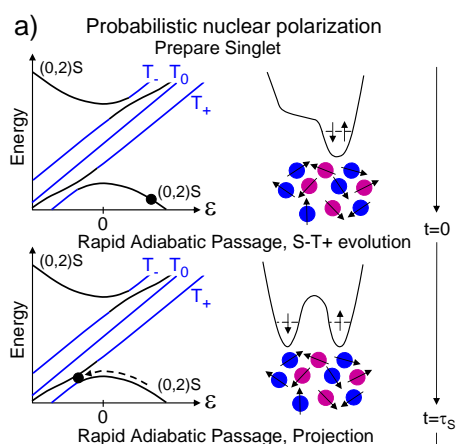

b) Adiabatic nuclear polarization
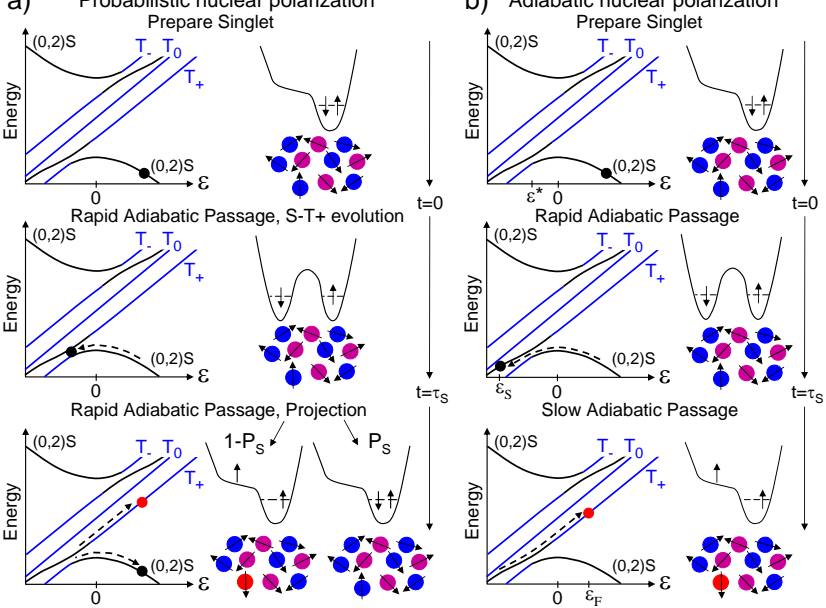

FIG. 2: (a) Probabilistic nuclear polarization sequence. (b) Adiabatic nuclear polarization sequence (see text).

being in the singlet state after time $\tau_{\mathrm{S}}$ thus appears as charge signal - the difference between the $(1,1)$ and $(0,2)$ charge states - detected by measuring the time averaged QPC conductance, $g_{\mathrm{S}}$ (see Fig. 1(a)). Figure 1(c) shows $g_{\mathrm{S}}$ as a function of $V_{\mathrm{L}}$ and $V_{\mathrm{R}}$ with this pulse sequence applied. The field dependence of this signal is shown in Fig. $1(\mathrm{~d})$, which plots the calibrated singlet state probability, $P_{S}$, as a function of $B_{0}$ and $\epsilon_{\mathrm{S}}$. In Fig. $1(\mathrm{~d}), P_{S} \sim 0.7$ at the $S-T_{+}$degeneracy, corresponding to a probability of electron-nuclear flip-flop per cycle $\left(1-P_{S}\right) \sim 0.3$. The position of the anti-crossing, $\epsilon_{\mathrm{S}}=\epsilon^{*}$, becomes more negative as $B_{0}$ decreases toward zero (Fig. $1(\mathrm{~d})$ ), as expected from the level diagram, Fig. 1(b).

An alternative sequence that (in principle) deterministically flips one nuclear spin per cycle and allows greater control of the steady-state nuclear polarization is shown in Fig. 2(b): In this case, the initial $(0,2) S$ is separated quickly $(\sim 1 \mathrm{~ns})$, to a value of detuning beyond the $S$ $T_{+}$resonance, $\epsilon_{\mathrm{S}}<\epsilon^{*}$. Since the time spent at the $S-T_{+}$ resonance during the pulse is short, the singlet is preserved with high probability. Next, detuning is brought back toward zero, to a value $\epsilon_{\mathrm{F}}$, on a time scale slow compared to $T_{2}^{*}(\sim 100 \mathrm{~ns})$. This converts $S$ to $T_{+}$and flips a nuclear spin each cycle. Detuning is then rapidly moved to the point $\mathrm{M}$ (again, over a time $\sim 1 \mathrm{~ns}$ ). While slightly different, both pulse sequences rely on bringing the $S$ and $T_{+}$states into resonance; the otherwise large difference between nuclear and electron Zeeman energies would prevent flip-flop processes [23. We now explore characteristics of the resulting nuclear polarization for each of the processes illustrated in Fig. 2.

We begin by examining the statistical polarization sequence shown in Fig. 2(a). We first measure $P_{S}$ as a function of $B_{0}$ and $\epsilon_{\mathrm{S}}$, with $\tau_{\mathrm{S}}=100 \mathrm{~ns}, \tau_{\mathrm{P}}=300 \mathrm{~ns}$, $\tau_{\mathrm{P}^{\prime}}=100 \mathrm{~ns}, \tau_{\mathrm{M}}=32 \mu \mathrm{s}$. These data, shown in Fig. 3(a), map out the $S-T_{+}$anti-crossing in the limit of minimal 

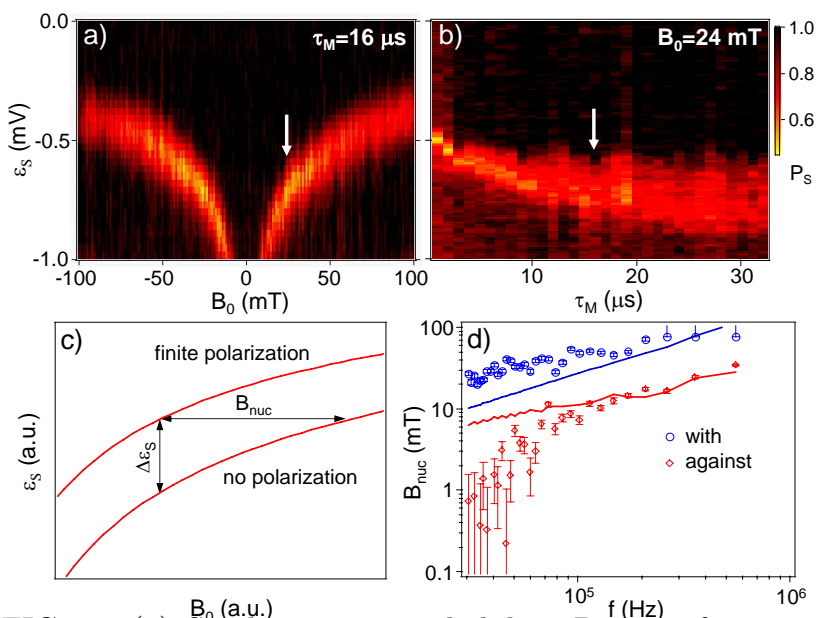

FIG. 3: (a) Singlet return probability $P_{S} \mathrm{f}$ as a function of applied field $B_{0}$ and detuning at the separation point, $\epsilon_{\mathrm{S}}$. (b) Dependence of the $S-T_{+}$anti-crossing position on measurement duration $\tau_{\mathrm{M}}$, with $B_{0}=24 \mathrm{mT}$. A decrease in $\tau_{\mathrm{M}}$ increases the polarization rate, shifting the $S-T_{+}$resonance condition to more positive $\epsilon_{\mathrm{S}}$. (c) Schematic diagram illustrating the effect of nuclear polarization on the spin funnel. Polarization increases $B_{\text {nuc }}$, shifting the funnel to more positive $\epsilon_{\mathrm{S}}$. The value of $B_{\text {nuc }}$ is extracted by measuring the shift in the $S-T_{+}$position relative to the position without polarization. (d) $B_{\text {nuc }}$ as a function of cycle frequency, $f$, measured sweeping $\epsilon_{\mathrm{S}}$ to increasing values ("with") and decreasing values ("against"). In the increasing sweep direction, the motion of $\epsilon_{\mathrm{S}}$ coincides with the polarization-induced motion of the $S$ $T_{+}$anti-crossing, resulting in a higher polarization. Solid lines are predictions from a nonlinear diffusion model (see text).

measurement induced polarization (polarization is negligible for $\tau_{\mathrm{M}}>30 \mu \mathrm{s}$, as will be shown below). A steadystate nuclear polarization at $B_{0}=24 \mathrm{mT}$ results in a shift in the position of $\epsilon^{*}$, depending on the measurement time $\tau_{\mathrm{M}}$, which determines the cycle period (Fig. 3b). As $\tau_{\mathrm{M}}$ decreases, the position of $\epsilon^{*}$ moves to larger values of detuning, indicating an increase in the average $B_{\text {nuc }}$. For $\tau_{\mathrm{M}}>30 \mu \mathrm{s}$ the value of $\epsilon^{*}$ saturates to its unpolarized value. Values for $B_{\text {nuc }}\left(\tau_{\mathrm{M}}\right)$ can be extracted from calibrating the shift in $\epsilon^{*}$ using the data in Fig. 3(a), as illustrated in Fig. 3(c).

We find that both the position, $\epsilon^{*}$, and the width of the region where $P_{S}\left(\epsilon_{\mathrm{S}}\right)$ is reduced below unity - marking the mean and fluctuations of the position of the $S-T_{+}$ anti-crossing - depend on the sweep direction used for data acquisition. In the present measurements, $\epsilon_{\mathrm{S}}$ is swept on the inner loop and $\tau_{\mathrm{M}}$ is increased on the outer loop. Sweeping $\epsilon_{\mathrm{S}}$ to more negative values (downward in Fig. 3(b)), i.e., against the movement of $\epsilon^{*}$ with increasing polarization, results in smaller nuclear polarization and a narrower resonance width compared to sweeping $\epsilon_{\mathrm{S}}$ to more positive values (not shown), i.e., with the motion of $\epsilon^{*}$ as polarization builds up. The extracted $B_{\text {nuc }}$ as a function of cycle frequency, $f=1 /\left(\tau_{\mathrm{P}}+\tau_{\mathrm{P}^{\prime}}+\tau_{\mathrm{S}}+\tau_{\mathrm{M}}\right)$ is shown in Fig. 3(d) for the two sweep directions of $\epsilon_{\mathrm{S}}$.
In both cases, polarization increases with $f$ reaching a maximum value of $B_{\text {nuc }} \sim 80 \mathrm{mT}$ (see Fig. 3(d)).

Qualitative features of the statistical DNP cycle (Fig. 2(a)) are accounted for by nonlinear rate equations for the nuclear polarization in the left and right dots, $P_{L, R}$. Within this model, a polarization-dependent probability per cycle of electron nuclear flip-flop, $p_{\text {flip }}(P)$, where $P=P_{L}+P_{R}$, induces nuclear polarization, while out-diffusion relaxes polarization with rates $\Gamma_{L}$ and $\Gamma_{R}$ in the left and right dots. Nonlinearity arises from the dependence of $p_{\text {flip }}$ on the distance $\Delta=\epsilon_{\mathrm{S}^{-}} \epsilon^{*}$ from the separation point to the anti-crossing, which in turn depends on $B_{\text {tot }}$, and hence $P$. The relevant energy scale for $\Delta$ is the width of the $S-T_{+}$anti-crossing, $\Omega$. Writing $\Omega=\hbar \sqrt{2} \cos (\theta) / T_{2}^{*}$, where $\theta=\arctan \left(\frac{2 t}{\epsilon_{\mathrm{S}}-\sqrt{4|t|^{2}+\left(\epsilon^{*}\right) 2}}\right)$ is the adiabatic angle 24] that accounts for charge state mixing near $\epsilon=0, T_{2}^{*}$ is the inhomogeneously broadened transverse relaxation time at the $S-T_{+}$anti-crossing, and $t$ is the interdot tunnel coupling, we find that the probability of a spin flip per cycle is given by $p_{\text {flip }}(P)=\left[\Delta^{2} /\left(2 \Omega^{2}\right)+2\right]^{-1}$. Note that $p_{\text {flip }}=1 / 2$ at the anti-crossing, $\epsilon_{\mathrm{S}}=\epsilon^{*}$. Polarizations $P_{L}, P_{R}$ of the left and right dots then evolve according to coupled diffusion equations,

$$
\frac{d P_{L, R}(t)}{d t}=-\Gamma_{L, R} P_{L, R}+\frac{p_{\text {flip }}(P) f N_{L, R}}{3 / 2\left[N_{L}+N_{R}\right]^{2}},
$$

where $N_{L}$ and $N_{R}$ are the number of nuclei in the left and right dots. The factor of $3 / 2$ reflects the spin- $3 / 2$ $\mathrm{Ga}$ and As nuclei. Results of the model using experimental parameters $N_{L} \sim N_{R} \sim 3 \times 10^{6}$, with $\Gamma=\Gamma_{L}+\Gamma_{R}$ as a fit parameter (assuming $\Gamma_{L}=\Gamma_{R}$ ) are shown in Fig. 3(d). The interdot tunnel coupling, $t /\left(g^{*} \mu_{B}\right) \sim 390 \mathrm{mT}$, is determined from measurements of the charge occupancy as a function of detuning near the $(1,1)-(0,2)$ interdot charge transition [25].

Qualitative agreement between experiment and model are seen in the dependence of polarization on cycle frequency and sweep direction. The fit gives $\Gamma \sim 0.3 \mathrm{~s}^{-1}$ which is consistent with out-diffusion predominantly perpendicular of the plane of the 2DEG, $\Gamma \sim D / d^{2}$, taking reasonable values for the electron gas thickness $d \sim 6 \mathrm{~nm}$ and the nuclear diffusion constant $D \sim 10^{-13} \mathrm{~cm}^{2} / \mathrm{s}[26$. Polarizations exceeding the $\sim 1 \%$ observed here can be achieved by increasing the cycle rate. Baugh et al. obtained polarizations approaching $20 \%$ in the Pauli blockade regime, which was associated with a tunneling rate of $10^{8} \mathrm{~Hz}$, several orders of magnitude faster than the polarization cycle frequencies employed here [12.

We next investigate a self-limiting adiabatic DNP cycle that allows steady-state nuclear polarization to be set by a gate voltage, as illustrated in Fig. 2(b). The cycle is shown in terms of motion along energy levels in Fig. 4(a) and motion within the charge stability diagram in the inset of Fig. 4(c). The new feature in this sequence, 

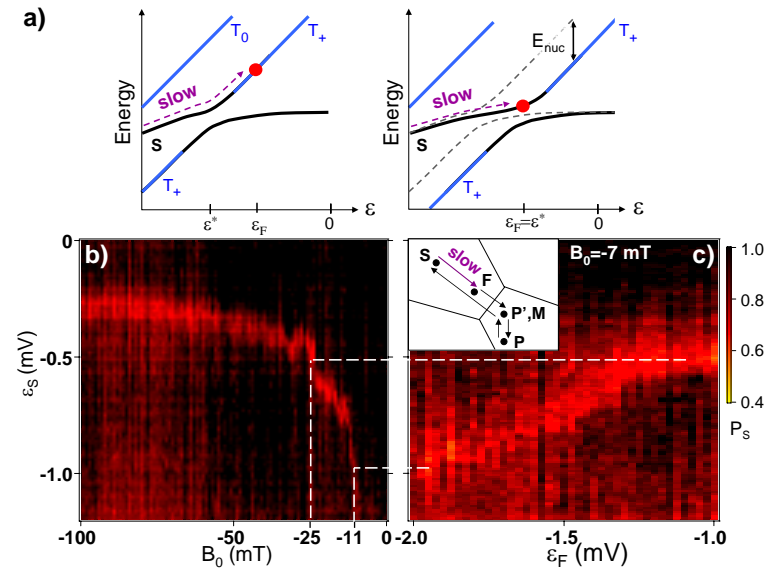

FIG. 4: (a) Self-limiting adiabatic polarization sequence. Left: Starting with $B_{\text {nuc }}=0$, the sequence polarizes nuclear spins and $B_{\text {nuc }}$ increases, shifting the $S-T_{+}$resonance to more positive $\epsilon$. Right: Steady state is reached when $\epsilon^{*}=\epsilon_{\mathrm{F}}$. Dashed grey lines show the energy level configuration before nuclear polarization. (b) Calibration spin funnel acquired using the pulse sequence (Fig. 1(c)). (c) $P_{S}$ as a function of $\epsilon_{\mathrm{F}}$ measured with the adiabatic polarization sequence applied. The complete pulse sequence consists of three adiabatic polarization cycles (a single adiabatic polarization cycle is shown in the inset) followed by one measurement cycle (shown in Fig. 1(c)). The combined pulse sequence length is $\sim 6.4 \mu \mathrm{s}$. Steady state hyperfine field $B_{\text {nuc }}$ changes from $4 \mathrm{mT}$ to 18 $\mathrm{mT}$ as set-point $\epsilon_{\mathrm{F}}$ is moved from $-2 \mathrm{mV}$ to $-1.3 \mathrm{mV}$.

compared to the statistical DNP cycle (Fig. 2(a)), is that the cycle passes through the anti-crossing at $\epsilon^{*}$ quickly during the pulse to the separation point $\epsilon_{\mathrm{S}}$, then slowly back to a point $\epsilon_{\mathrm{F}}$, before moving quickly to the measurement point, $\epsilon_{\mathrm{M}}$. The cycle then continues through $\epsilon_{\mathrm{P}}$ and $\epsilon_{\mathrm{P}^{\prime}}$ as before. The position of $\epsilon_{\mathrm{F}}$ is chosen so that at low polarization it is to the right of $\epsilon^{*}$, as seen in the left diagram in Fig. 4(a). Adiabatic crossing of the $S-T_{+}$ anti-crossing in the return direction results in a single electron-nuclear flip-flop and leads to nuclear polarization upon cycling. As polarization builds, $\epsilon^{*}$ moves to more positive values of detuning, until it coincides with the set-point, $\epsilon_{\mathrm{F}}$, as illustrated on the right panel of Fig. 4(a). At that point, the build-up of polarization stops.

To both induce and measure a steady-state nuclear polarization, a sequence of three DNP cycles (Fig. 4(c), inset) followed by one measurement cycle (Fig. 1(c)) is iterated. We first calibrate changes in the position along $\epsilon$ with changes in $B_{\text {nuc }}$ by measuring the singlet return probability $P_{S}$ as a function of $\epsilon_{\mathrm{S}}$ and applied field $B_{0}$, showing how $\epsilon^{*}$ depends on $B_{\text {tot }}$ in the absence of nuclear polarization (Fig. 4(b)). Figure 4(c) shows that for the specific case of $B_{0}=7 \mathrm{mT}$, the steady-state nuclear polarization can be controlled by the position of the set-point $\epsilon_{\mathrm{F}}$, ranging from $B_{\mathrm{nuc}} \sim 4 \mathrm{mT}$ for $\epsilon_{\mathrm{F}}=-2 \mathrm{mV}$ to $B_{\text {nuc }} \sim 18 \mathrm{mT}$ for $\epsilon_{\mathrm{F}}=-1.3 \mathrm{mV}$. For $\epsilon_{\mathrm{F}}>-1.3 \mathrm{mV}, B_{\text {nuc }}$ saturates at $\sim 18 \mathrm{mT}$. In these measurements, the low fields involved were necessary to allow calibration using plots like Fig. 4(a), which flatten and become insensitive to changes in $B_{\text {nuc }}$ at higher fields. This can be overcome by using single-electron spin resonance [15, 27, which allows calibration of $B_{\text {nuc }}$ at arbitrary fields. Polarization with the adiabatic pulse sequence is less than expected given the cycle rate (it should be twice as efficient as the probabilistic pulse sequence at a given cycle frequency, $f$ ) and did not grow when the cycle frequency was increased beyond the value used to acquire the data in Fig. 4(c). Further experiments are required to determine if dark state formation is limiting polarizations obtained with this pulse sequence, where successive interactions at the S- $T_{+}$degeneracy take place on $\sim 300$ ns time scales, which are much shorter than nuclear spin decoherence times [23].

This work was supported by DARPA, DTO, NSFNIRT (EIA-0210736), Harvard Center for Nanoscale Systems, and Princeton University. Research at UCSB supported in part by QuEST, an NSF Center.

* These authors contributed equally.

[1] L. P. Kouwenhoven et al., in Mesoscopic Electron Transport (eds L. P. Kouwenhoven, G. Schön, and L. L. Sohn, Kluwer, Dordrecht, (1997).

[2] R. Hanson et al., Rev. Mod. Phys. (in press), arXiv:condmat/0610433. (2006).

[3] G. Chen, D. L. Bergman, L. Balents, cond-mat/0703631.

[4] W. A. Coish et al., J. Appl. Phys. 101, 081715 (2007).

[5] H. Christ, J. I. Cirac, G. Giedke, Phys. Rev. B 75, 155324 (2007).

[6] M. S. Rudner, L. S. Levitov, cond-mat/0705.2177.

[7] K. Wald et al., Phys. Rev. Lett. 73, 1011 (1994).

[8] G. Yusa et al., Nature 434, 1001 (2005).

[9] K. Ono et al., Science 297, 1313 (2002).

[10] F. H. L. Koppens et al. Science 309, 1346 (2005).

[11] K. Ono, S. Tarucha, Phys. Rev. Lett. 92, 256803 (2004).

[12] J. Baugh et al., cond-mat/0705.1104.

[13] A. C. Johnson et al., Nature 435, 925 (2005).

[14] J. R. Petta et al., Science 309, 2180 (2005).

[15] F. H. L. Koppens et al., Nature 442, 766 (2006).

[16] S. I. Erlingsson, Y. V. Nazarov, V. I. Falko, Phys. Rev. B 64, 195306 (2001).

[17] A. V. Khaetskii, D. Loss, L. Glazman, Phys. Rev. Lett. 88, 186802 (2002).

[18] D. Klauser, W. A. Coish, D. Loss, Phys. Rev. B 73, 205302 (2006).

[19] D. Stepanenko et al., Phys. Rev. Lett. 96, 136401 (2006).

[20] J. M. Taylor, C. M. Marcus, M. D. Lukin, Phys. Rev. Lett. 90, 206803 (2003).

[21] M. V. G. Dutt et al., Science 316, 1313 (2007).

[22] D. Paget, G. Lampel, B. Sapoval, Phys. Rev. B 15, 5780 (1977).

[23] A. Imamoglu et al., Phys. Rev. Lett. 91, 017402 (2003).

[24] J. M. Taylor et al., Phys. Rev. B 76, 035315 (2007).

[25] L. DiCarlo et al., Phys. Rev. Lett. 92226801 (2004).

[26] D. Paget, Phys. Rev. B 25, 4444 (1982).

[27] E. A. Laird et al. arXiv:0707.0557 (2007); K. C. Nowack 
et al. arXiv:0707.3080 (2007). 